\title{
El papel de la asertividad docente en el desa- rrollo de la competencia social de su alumnado
}

\author{
Villena Martínez, M.D., Justicia Justicia, F. y \\ Fernández de Haro, E.
}

Departamento de Psicología Evolutiva y de la Educación

Universidad de Granada

\section{España}

Correspondencia: $M^{a}$ Dolores Villena Martínez. Departamento de Psicología Evolutiva y de la Educación. Facultad de Ciencias de la Educación. Campus Universitario de Cartuja s/n. E-18071. Granada. España. E-mail: dvillena@ugr.es

(C) Education \& Psychology I+D+i and Ilustre Colegio Oficial de la Psicología de Andalucía Oriental (Spain) 


\section{Resumen}

Introducción. La competencia social del alumnado ha sido ampliamente estudiada desde la perspectiva de los efectos que produce en el niño ser o no socialmente competente, mientras que ha sido escaso su análisis desde la contribución del maestro a su desarrollo. El objetivo de esta investigación es valorar la influencia de la asertividad del docente en la competencia social de sus alumnos y en la cohesión del grupo-clase.

Método. La muestra estuvo constituida por 36 profesores y 302 alumnos de sexto curso de Primaria. Los docentes cumplimentaron el cuestionario ADCAs. Autoinformes de Actitudes y Valores en las Interacciones Sociales, en su forma ADCA-pr y los estudiantes el AECS. Actitudes y Estrategias Cognitivas Sociales y la forma A del BULL-S. Test de Evaluación de la Agresividad entre Escolares. En un primer análisis de regresión lineal se emplearon como variables dependientes las actitudes que comprenden la competencia e incompetencia social. Las variables independientes fueron la autoasertividad, heteroasertividad, edad y sexo del docente, así como la edad y sexo del alumno como moduladoras de dicho efecto. Por otro lado, se empleó un modelo de Estimación de Ecuaciones Generalizadas en el que la variable dependiente fue la cohesión de grupo y las independientes la autoasertividad, heteroasertividad, edad y sexo del docente.

Resultados. Los resultados muestran, por un lado, que la heteroasertividad del maestro afecta a la competencia social del alumnado amortiguando su dominancia (actitud antisocial), y por otro, que tanto la autoasertividad como la heteroasertividad influyen en la cohesión del grupo, aunque dicho efecto está mediado por el género del docente y por el propio desarrollo de este constructo.

Discusión y conclusiones. Se confirma que la asertividad influye positivamente en el desarrollo de la competencia social de los alumnos, específicamente en la de aquellos que manipulan y se aprovechan de los otros en beneficio propio sintiéndose superiores a ellos, al tiempo que aumenta la cohesión entre los miembros del grupo-clase. Se configura, así, como una competencia interpersonal deseable en el perfil profesional docente.

Palabras Clave: Asertividad, competencia social, cohesión del grupo, relaciones interpersonales, primaria. 


\title{
The role of teacher assertiveness in the development of social competence of their students
}

\begin{abstract}
Introduction. The social competence of school students has been studied extensively in terms of the effect on children of being or not being socially competent. However, there has been little analysis of how teachers contribute to the development of these skills. This research assesses the influence of teacher assertiveness on the social competence of their students and on the cohesion of the class group.

Method. The sample consisted of 36 teachers and 302 sixth-year Primary School students. The teachers filled in the ADCAs Self-assessment Report on Attitudes and Values in Social Interactions in the ADCA-pr form, and the students the AECS Social Attitudes and Cognitive Strategies report and form A of the BULL-S Inter-Pupil Aggressiveness Assessment Test. A first linear regression analysis took as dependent variables the attitudes comprising social competence and incompetence. The independent variables were the self-assertiveness, hetero-assertiveness, age and gender of the teacher, and the age and gender of the student as modulators of the effect. We also used a Generalised Estimating Equation model in which the dependent variable was the cohesion of the group and the independent variables were the self-assertiveness, hetero-assertiveness, age and gender of the teacher.

Results. The results show, on one hand, that the teacher's hetero-assertiveness affects students' social competence, lessening their dominance (antisocial attitude), and on the other, that both selfassertiveness and hetero-assertiveness influence the cohesion of the group, although this effect is mediated by the gender of the teacher and the construct itself.

Discussion and Conclusion: It is confirmed that assertiveness positively influences the development of students' social competence, and more specifically, that of students who manipulate and take advantage of others for their own benefit and feel themselves to be superior to them, while increasing cohesion among the members of the class group. Therefore, it appears to be a desirable interpersonal skill for professional teachers.
\end{abstract}

Keywords: assertiveness, social competence, group cohesion, interpersonal relationships, primary school. 


\section{Introducción}

El conocimiento sobre la realidad social, es decir, sobre las instituciones, las normas, valores, personas y las relaciones que se establecen entre ellas y que ordenan su correcto funcionamiento, se configura como una pieza clave para la integración del niño en la sociedad y para su formación como adulto socialmente adaptado. Desde hace un par de décadas dicha realidad ha cambiado como consecuencia de la influencia de variables sociales, políticas y económicas, y la sociedad se ha visto abocada a numerosas y rápidas transformaciones. De manera paralela a este proceso, y en ocasiones como resultado del mismo, los jóvenes parecen haber recibido una socialización que no les ha capacitado para hacer frente a dichas demandas. En la búsqueda de comprensión y adaptación a tales circunstancias se han visto forzados a modificar un elemento clave para el desarrollo social, su jerarquía de valores.

Uno de los enfoques más recientes en el estudio de los valores los considera fruto de este conjunto de variables (Ros, 2001a), es decir productos culturales que como tales cogniciones facilitan el encuentro entre el sujeto y la sociedad (Braithwaite y Blamey, 2001). Dicho ajuste viene dado por la relación existente entre éstos y las conductas. Desde la perspectiva psicosocial, la importancia de los valores reside ahí, en su capacidad para orientar y dirigir el comportamiento de los sujetos (Pérez, 2009). Se definen, por tanto, como "creencias jerarquizadas sobre estilos de vida y formas de existencia que guían nuestras actitudes y comportamientos" (Ros, 2001b, p.85).

Con independencia de la diversidad y carácter jerárquico, los valores sociales se consideran universalmente deseables. Son uno de los instrumentos más efectivos del sistema para la transmisión del conocimiento social (Hernández, 1986) y están dirigidos a promover la convivencia pacífica en una sociedad democrática y plural. Siendo esta finalidad uno de los cuatro aprendizajes fundamentales en torno a los cuales debe estructurarse la educación (Delors, 1996), adquieren una relevancia destacada en este ámbito. Atendiendo a ello, y dado que la escuela es uno de los principales entornos de socialización, el centro educativo se configura como un espacio privilegiado donde aprenderlos y practicarlos, ya que para que pueda tener lugar la apropiación de un valor es indispensable la experiencia del mismo (Ortega y Mínguez, 2001). De forma similar sucede en el aula, donde las relaciones entre sus miembros vienen determinadas por las competencias sociales de que disponen. Unas relaciones que retro- 
alimentan no sólo los comportamientos sino también las emociones y pensamientos, contribuyendo a la generación del ambiente social de la clase (Bueno y Garrido, 2012). Así lo pusieron de manifiesto Allen, Weissberg y Hawkins (1989) en los comienzos de la investigación en valores, al constatar su vinculación con la competencia social en la adolescencia temprana. Los autores determinaron que los valores sociales podían mediar en la influencia que tienen los iguales sobre el individuo, inhibiendo o facilitando la realización de conductas que, en último término, frenen su proceso de adaptación social.

Según esto, se puede afirmar que tanto las circunstancias sociales actuales como la evidencia empírica han contribuido a que la formación en valores en los contextos escolares sea regulada normativamente. Sin embargo, dicha regulación, ya se desarrolle de manera transversal o con currículo propio, no garantiza el aprendizaje de los mismos por parte del alumnado. Este objetivo puede verse truncado cuando los valores intencionalmente promovidos por el centro no coinciden con los expresados por la conducta del docente, el cual actúa y educa desde su concepción del mundo y su propio sistema de valores (Díaz, 2006; Gómez, 2005; Ortega y Mínguez, 2001) al amparo del "hábito del profesor isla" (Fernández, 1998, p.38).

La idea de doble mensaje alcanza especial relevancia al considerar que el maestro constituye un modelo a seguir por el alumnado (Delval y Enesco, 1994; Muñiz y Pérez, 2010) y que el aprendizaje observacional hace posible la adquisición no sólo de pautas específicas de comportamiento social sino de actitudes y estrategias (Rivière, 1992). Concretamente, se estima que es uno de los principales modelos a través de los cuales se desarrolla la competencia social en los niños (Corredor, Justicia-Arráez, Pichardo y Justicia, 2013).

Habilidades sociales, conducta asertiva y competencia social han sido habitualmente empleados como sinónimos. Bueno y Garrido (2012) entienden que las habilidades interpersonales constituyen un caso concreto de relaciones sociales, al tiempo que las reconocen equivalentes a las habilidades sociales. Las definiciones formuladas en torno a estas últimas coinciden en concebirlas como comportamientos aprendidos (habilidades) que tienen lugar en el contexto de las relaciones interpersonales (sociales), que están orientados a conseguir unos objetivos concretos y regulados por las reglas sociales específicas de los contextos culturales y situacionales. 
Desde un punto de vista cognitivo-conductual integrado, García y Magaz (2000) delimitan la conducta asertiva como la clase de conductas o habilidades sociales (Caballo, 2005; Gismero, 1996; Pérez, 2000) que constituyen un acto de respeto por igual a uno mismo y a las personas con quienes se interacciona. Así, una persona se comporta asertivamente al mostrar la conducta social apropiada en el momento oportuno. Cuando las personas del entorno valoran dicha conducta como adecuada se hace referencia a su competencia social, ya que ésta se encuentra vinculada a la calidad de la ejecución de la conducta en función del contexto (García, Sureda y Monjas, 2010; Mcloughlin, 2009; Trianes, De la Morena y Muñoz, 1999). Según esto, en la medida en que la persona asertiva es percibida como socialmente competente ambos términos se consideran sinónimos (Adams y Roopnarine, 1994; Bueno y Garrido, 2012 y Delamater y McNamara, 1991), aunque este último concepto pueda tener un carácter más amplio y abierto.

De las múltiples definiciones de competencia social que se ofrecen, en este trabajo se adopta la desarrollada por Moraleda, González y García-Gallo (1998). Los autores crearon un modelo teórico desde el que la conciben como la expresión de ciertas actitudes básicas y determinados procesos cognitivos que darán lugar al éxito o al fracaso en las relaciones sociales y que, en definitiva, facilitarán u obstaculizarán la adaptación social al medio. En el medio escolar son numerosas las investigaciones que informan que la competencia social es un elemento clave en la adaptación de los alumnos a la escuela (Blankemeyer, Flannery y Vazsonyi, 2002; Ladd, 1990; Monjas y González, 1998; Muñoz, Carreras y Braza, 2004; Ollendick, Francis y Baum, 1991), facilitando la calidad en las relaciones interpersonales y previniendo comportamientos delictivos y conductas agresivas (Corapci, 2008; Frey, Hirschstein y Guzzo, 2000; Trianes, 1996). También se han observado relaciones positivas entre la competencia social y algunos ámbitos del desarrollo personal directamente implicados en las relaciones interpersonales, tales como la empatía (Monjas, 2002) o el autocontrol (Barr, Kahn y Schneider, 2008).

\section{Objetivos e hipótesis}

En la ctualidad no se cuestiona la importancia que tiene para el desarrollo personal y social del niño el poseer una adecuada competencia social. Sin embargo, los estudios continúan poniendo más énfasis en indagar dicha relación que en estudiar los factores del contexto escolar que la determinan. 
Aunque la figura del docente se ha analizado ampliamente en lo concerniente a la importancia de las expectativas, empatía, amabilidad y entusiasmo, liderazgo o su estilo de enseñanza (Trianes et al., 1999), no se ha profundizado en el conocimiento de los efectos que su competencia social puede tener sobre la del alumnado (Muñoz y Jiménez, 1995). Analizar la aportación del maestro a ello, a través del estilo relacional que tiene con sus alumnos, será el objetivo de estudio de esta investigación. En este trabajo se valora su contribución en dos niveles: en el alumno individualmente considerado, a través de sus actitudes sociales, y en las relaciones que en el grupo-clase se generan, mediante la denominada cohesión grupal, ya que ésta ayuda a que "sus miembros desarrollen valores como: sentido de lealtad, autoestima, seguridad, etc.” (Oñate, 1998, p.549).

En este contexto, se plantean las siguientes hipótesis:

H1. Los niños de los centros privados-concertados tendrán mejores actitudes sociales que los escolarizados en colegios públicos.

El género del docente influirá en el desarrollo de las actitudes sociales del alumnado, afirmación desde la cual se establece que:

H2. Las mujeres favorecerán el desarrollo de actitudes prosociales.

H3. Los hombres promoverán el desarrollo de actitudes antisociales y asociales

Con respecto a la edad del docente, se espera comprobar que:

H4. Los maestros de mayor edad suscitarán en su alumnado actitudes prosociales.

H5. Los maestros de menor edad contribuirán a que sus alumnos tengan actitudes antisociales y asociales.

Respecto a la asertividad del docente:

H6: Los maestros autoasertivos promoverán en su alumnado actitudes prosociales.

H7. Los maestros autoasertivos frenarán el desarrollo de actitudes antisociales y asociales en su alumnado.

H8. Los maestros heteroasertivos promoverán en su alumnado actitudes prosociales.

H9. Los maestros heterooasertivos frenarán el desarrollo de actitudes antisociales y asociales en su alumnado.

H10. Los docentes asertivos generarán una buena cohesión entre los miembros del grupo. 


\section{Método}

\section{Participantes}

Formaron parte del estudio dos grupos de sujetos vinculados por la relación profesoralumno. Por un lado, 36 profesores, 15 hombres (41.7\%) y 21 mujeres (58.3\%), con una edad media de 45.47 años $(D . T .=10.97$, rango $=24-60)$. La media de horas por semana que pasaban con sus alumnos fue de aproximadamente 5 horas $(D . T .=5.39$, rango $=1-18.50)$. Por otro lado, un total de 302 alumnos de $6^{\circ}$ curso de Educación Primaria Obligatoria, de los cuales 148 eran niños $(49 \%)$ y 154 niñas $(51 \%)$, con una edad media de 11.62 (D.T. $=.56$, rango $=$ 11-14), situándose los mayores porcentajes entre los 11 (41.2\%) y 12 años (55.5\%).

La selección de los colegios participantes se hizo atendiendo a la ubicación de los mismos, al nivel socioeconómico y cultural de su alumnado, al carácter público o privado y, en última instancia, al interés del profesorado por colaborar en la investigación. Con estos criterios, se eligieron ocho colegios de la provincia de Granada (España): cuatro de la capital, dos pertenecientes a la zona centro, con carácter concertado y alumnado con nivel socioeconómico medio-alto y alto, y dos a la periferia, con carácter público y nivel medio-bajo y bajo. Los otros cuatro centros, dos estaban situados en el extrarradio y dos en diferentes pueblos de la provincia; todos de carácter público y con alumnado de nivel socioeconómico variado.

\section{Instrumentos}

Para la recogida de datos se utilizaron tres cuestionarios elaborados y validados con muestras españolas:

ADCAs. Autoinformes de Actitudes y Valores en las Interacciones Sociales (García y Magaz, 2000). Cumplimentado por el profesorado en su forma ADCA-pr, pretende identificar sus actitudes y valores respecto de las relaciones sociales que mantiene con su alumnado. Se basa en el constructo de asertividad, definido por los autores como «clase de conductas sociales que constituyen un acto de respeto por igual a uno/a mismo/a y a las personas con quienes se desarrolla la interacción» (p. 13). Para evitar la compensación de puntuaciones entre los componentes, se divide en dos subescalas. De un lado, con 20 ítems y un coeficiente de fiabilidad para la muestra de $\alpha=.776$, la auto-asertividad (AA), con la que se mide el grado con el que una persona se respeta a sí misma: y por otro, con 15 ítems y un índice $\alpha=.869$, la hete- 
ro-asertividad (HA), grado en que respeta a los demás. En ambos casos se utiliza un sistema de respuesta de 4 opciones (Nunca o casi nunca, a veces, a menudo, siempre o casi siempre).

AECS. Actitudes y Estrategias Cognitivas Sociales (Moraleda et al., 1998). El cuestionario identifica las variables que facilitan o dificultan la adaptación social del sujeto y que conforman su competencia social. Dichas variables, actitudinales (Actitudes sociales) y de pensamiento (Pensamiento social), se presentan organizadas en una estructura bipolar en torno a la competencia social. Las Actitudes sociales, a su vez, se dividen en tres factores que contienen a otros de orden inferior. Así, el factor Prosocial o facilitador de las relaciones, con un coeficiente de fiabilidad para la muestra de $\alpha=.8$, incluye (1) la Conformidad con lo que es socialmente correcto con 9 ítems que valoran el acatamiento a las reglas y normas sociales que facilitan la convivencia y respeto mutuo, (2) Sensibilidad social, con 8 ítems que evalúan la tendencia del sujeto a sintonizar con los sentimientos ajenos, (3) Ayuda y colaboración con 10 ítems que miden la tendencia a participar y colaborar en el trabajo común, (4) Seguridad y firmeza en la interacción, con 10 ítems que analizan la confianza en las propias posibilidades para conseguir los objetivos de la interacción, y (5) Liderazgo prosocial, con 4 ítems que valoran la tendencia a aunar a los miembros del grupo en torno a unos objetivos comunes y a tomar la iniciativa. El factor denominado Antisocial o destructor de las relaciones, con un coeficiente de fiabilidad para la muestra de $\alpha=.642$, engloba (1) Agresividad-terquedad, que con 8 ítems evalúa la tendencia a la expresión violenta contra personas o cosas y a la tenacidad rígida como forma de agresividad, y (2) Dominancia, con 6 ítems que miden la tendencia a dominar a los demás para conseguir el propio provecho y a manipularles y aprovecharse de ellos. Por último, el factor Asocial o inhibidor de las relaciones, con un coeficiente de fiabilidad para la muestra de $\alpha=.562$, comprende (1) Apatía-retraimiento, que con 9 ítems analiza el desinterés de la persona por integrarse en los grupos y participar en sus actividades y la tendencia a mostrarse aislado, y (2) Ansiedad-timidez, que mediante 7 ítems estima la propensión a manifestar miedo a expresarse, a relacionarse y defender los propios derechos con asertividad. El bloque del Pensamiento Social, con una estructura similar, incluye tres factores, relacionados con el Estilo cognitivo, la Percepción social y el tipo de Estrategias cognitivas empleadas para problemas sociales. El cuestionario queda conformado por 137 ítems, a los que el alumnado debe responder con una escala tipo Likert con siete alternativas de respuesta que fluctúan desde (1) "no se da nada en ti" hasta (7) "se da muchísimo en ti”. Atendiendo al objetivo del estudio, se ha utilizado sólo la información concerniente a las Actitudes sociales. 
BULL-S. Test de Evaluación de la Agresividad entre Escolares (Cerezo, 2000). Analiza el acoso entre escolares desde la perspectiva de los diferentes agentes que intervienen en el aula al tiempo que ofrece información sobre variables de carácter grupal (ie. situación sociométrica y grado de cohesión). Presenta dos formas de aplicación con 15 preguntas cada una. La Forma A, para el alumnado, incluye tres dimensiones: la primera, con 4 ítems, relativa a la estructura socio-afectiva del grupo en su conjunto y de cada uno de sus miembros en particular; y la segunda y tercera informan sobre aspectos específicos de la relación agresor-víctima. Con la forma $\mathrm{P}$, de uso individual para el profesorado, se pretende que éste indique la situación de sus alumnos en la dinámica del bullying. En esta investigación se ha empleado la forma A, haciendo uso sólo de la información relativa a la cohesión grupal.

\section{Procedimiento}

Una vez seleccionados los colegios, se mantuvo un primer contacto con los directores de los centros para explicarles el objetivo de la investigación y solicitar el acceso al personal del centro para llevarla a cabo. Éstos convocaron una reunión con los posibles maestros participantes, a los que igualmente se les informó sobre la finalidad de la misma y en qué consistiría su colaboración. Se les hizo saber sobre la confidencialidad de los datos y su uso. Una vez que aceptaron formar parte, con cada equipo educativo se acordó el momento para pasar los cuestionarios al alumnado, ya que se trató de una administración colectiva en la que el investigador estaba presente. Por otra parte, a los docentes se les entregó el cuestionario ADCA-pr y se concretó un día para recogerlo.

\section{Análisis de datos}

Para estudiar la influencia de las actitudes sociales que tiene el profesorado cuando interacciona con su alumnado en el desarrollo de la competencia social de éste, se utilizaron modelos estadísticos lineales de regresión y análisis multivariante de la varianza dada la existencia de más de una variable dependiente correlacionadas entre sí (Steven, 2002). Como tal, se han tomado las variables del alumnado incluidas en el bloque de actitud social, con la agrupación de factores propuestos en el cuestionario. La edad y sexo de éste se consideraron variables independientes moduladoras de dicho efecto junto con la edad, sexo y grado de AA y de HA del maestro en función del número de horas que pasaba con su alumnado. Esta ponderación, realizada para determinar el peso de las variables, se debe a que los docentes interaccionaban con sus alumnos, en clase, un número de horas que variaba en función de la canti- 
dad y tipo de materias que les impartiesen. De este modo, se utilizó la puntuación media por hora que el maestro estaba con el alumnado. Destacamos el hecho de que las puntuaciones en AA y HA se estandarizaron a una escala común de 0 a 100 para que fuesen interpretables y comparables entre sí. Para la variable sexo del maestro se utilizó el de aquellos que más tiempo estaban con el grupo de alumnos.

Una vez comprobada la normalidad de las escalas de actitud mediante los gráficos $Q$ $Q$ se procedió a la determinación de los modelos estadísticos paramétricos, basados en supuestos normales, que mejor explicasen la relación de las variables independientes con la actitud social del alumno. El análisis de dichas relaciones, dado que hay más de una en el estudio, se realizó con la prueba estadística multivariante (MANOVA) Traza de Pillai ( $V$ ) ya que es la que ofrece una mayor potencia y robustez para detectar diferencias reales bajo distintas condiciones (Field, 2005).

Tras un primer modelo multivariante, donde se incluyen todas las variables de interés, se desestiman aquellas que no muestran relación significativa. Con las que sí la hay se establece un nuevo modelo reducido para analizar cómo se comportan a nivel individual, es decir, cómo influyen sobre las variables dependientes. Para cada modelo se verifican los supuestos estadísticos de aplicación sobre la igualdad en las matrices de covarianza con la prueba estadística de Box, por la cual se rechaza la hipótesis de igualdad de las matrices si el p-valor asociado es menor de .05. Finalmente, se verifica el buen ajuste del modelo con el estudio de normalidad de los residuos, indicando que si éstos se distribuyen según una curva normal el modelo ajustado será apropiado.

Con el objetivo de estudiar la relación entre la actitud social que tiene el profesor cuando interacciona con sus alumnos y la cohesión que existe entre éstos, se ajustaron diferentes modelos de Estimación de Ecuaciones Generalizadas (EEG) (Twisk, 2007) para la variable cohesión (variable binaria: 0 - baja o muy baja y 1 - buena). En dichos modelos se han considerado dos grupos de sujetos, alumnos y profesores, y un efecto intrasujeto que viene determinado por la clase/aula a la que el alumno pertenece. Para corregir el efecto entre sujetos, por pertenecer a una misma clase, derivado de la variable respuesta, se utiliza una matriz de covarianza sin estructura; estableciendo, igualmente, el nivel de significación al 5\%. 
Los datos se han analizado con el Statistical Package for the Social Sciencies (SPSS), versión 19.

\section{Resultados}

Respecto a las puntuaciones del cuestionario ADCA-pr, relativo a la medición de las actitudes y valores de los profesores en las interacciones sociales que mantienen con sus alumnos, la media de las puntuaciones estandarizadas se situó en 76.7 para la AA $(D . T .=$ 12.29) y 57.7 para la HA (D.T.= 16.64), existiendo una correlación moderada entre ellas dado que el coeficiente de correlación de Pearson fue de .521 .

Con relación a las puntuaciones obtenidas por el alumnado en el test AECS, usado para medir las variables que influyen en el grado de adaptación social de los sujetos al medio en que viven, en la Tabla 1 se muestran los estadísticos descriptivos.

Tabla 1. Medidas descriptivas del componente Actitud Social

\begin{tabular}{|c|c|c|c|c|c|c|}
\hline & Factor & Factor inferior & Media & D.T. & Mínimo & Máximo \\
\hline \multirow{5}{*}{$\begin{array}{l}\text { Competencia } \\
\text { Social }\end{array}$} & \multirow{5}{*}{ Prosocial } & Conformidad & 46.24 & 8.08 & 21 & 63 \\
\hline & & Sensibilidad social & 46.00 & 7.23 & 17 & 56 \\
\hline & & Ayuda y colaboración & 54.74 & 8.83 & 24 & 70 \\
\hline & & Seguridad y firmeza & 49.85 & 9.35 & 23 & 70 \\
\hline & & Liderazgo & 18.39 & 4.64 & 5 & 28 \\
\hline \multirow{4}{*}{$\begin{array}{l}\text { Incompetencia } \\
\text { Social }\end{array}$} & \multirow{2}{*}{ Antisocial } & Dominancia & 17.36 & 6.71 & 6 & 39 \\
\hline & & Agresividad-terquedad & 25.61 & 8.01 & 8 & 56 \\
\hline & \multirow{2}{*}{ Asocial } & Apatía-retraimiento & 26.51 & 8.04 & 9 & 52 \\
\hline & & Ansiedad-timidez & 24.99 & 8.05 & 7 & 48 \\
\hline
\end{tabular}

Se comprobó si existían diferencias significativas en las actitudes sociales de los alumnos en función del tipo de centro al que perteneciesen, público o concertado. Para ello se realizó la prueba t-Student, que arrojó datos de igualdad de medias para ambas muestras. Es por esto que se procedió a analizar los datos sin desglosar por tipo de centro.

Para las actitudes sociales se analizaron, pues, dos modelos de regresión lineal, uno para cada bloque:

\section{Actitud: Competencia Social}


En este grupo se incluyeron como variables dependientes los factores de Conformidad con lo socialmente correcto, Sensibilidad social, Ayuda y colaboración, Seguridad y firmeza en la interacción y Liderazgo prosocial. Una vez comprobado el grado de correlación entre ellos, donde la correlación de Pearson estaba comprendida entre .5 y .7, se generó un modelo multidimensional en el que se encontró que ni la edad, ni el sexo del alumno, ni la edad, sexo, grado de AA y grado de HA del profesor mostraban relación significativa con los factores de Liderazgo y Seguridad y firmeza, por lo que dichas variables dependientes fueron excluidas del modelo final. De este modo, el modelo que mejor explica qué afecta a la actitud prosocial del alumno (variable Sensibilidad social, Ayuda y colaboración y Conformidad con lo socialmente correcto) viene determinado por su sexo $(V=.068, F(3,297)=7.169, p<.001)$ y edad $(V=.027, F(3,297)=2.705, p<.05)$. Los resultados del modelo de regresión ajustado (Tabla 2) indican, con relación al sexo, que los niños tienen una tendencia a ser menos Sensibles socialmente que las niñas, que muestran menor grado de Ayuda y colaboración y que se manifiestan menos Conformes que éstas con lo que socialmente es correcto. Con relación a la edad, se observa que por cada año que aumenta la edad del alumnado disminuye en 2.11 su grado de Conformidad con lo que socialmente es correcto.

Tabla 2. Modelo de regresión para la Competencia Social

\begin{tabular}{|c|c|c|c|c|c|c|c|}
\hline \multirow[b]{2}{*}{ Variable dependiente } & \multirow[b]{2}{*}{ Parámetros } & \multirow[b]{2}{*}{ B } & \multirow[b]{2}{*}{ Error típ } & \multirow[b]{2}{*}{$\mathrm{t}$} & \multirow[b]{2}{*}{$\mathrm{p}$} & \multicolumn{2}{|c|}{$\begin{array}{c}95 \% \text { Intervalo de } \\
\text { confianza } \\
\end{array}$} \\
\hline & & & & & & $\begin{array}{l}\text { Límite } \\
\text { inferior }\end{array}$ & $\begin{array}{l}\text { Límite } \\
\text { superior }\end{array}$ \\
\hline \multirow[t]{3}{*}{ Sensibilidad social } & Constante & 53.888 & 8.198 & 6.573 & .000 & 37.755 & 70.020 \\
\hline & Sexo alumno varón vs mujer & -3.723 & .807 & -4.612 & .000 & -5.311 & -2.134 \\
\hline & Edad alumnado & -.523 & .707 & -.739 & .461 & -1.914 & .869 \\
\hline \multirow[t]{3}{*}{ Ayuda y colaboración } & Constante & 72.381 & 10.159 & 7.125 & .000 & 52.389 & 92.374 \\
\hline & Sexo alumno varón vs mujer & -3.177 & 1.000 & -3.176 & .002 & -5.145 & -1.208 \\
\hline & Edad alumnado & -1.387 & .876 & -1.582 & .115 & -3.111 & .338 \\
\hline \multirow{3}{*}{$\begin{array}{l}\text { Conformidad con lo } \\
\text { que es socialmente } \\
\text { correcto }\end{array}$} & Constante & 71.681 & 9.309 & 7.700 & .000 & 53.361 & 90.000 \\
\hline & Sexo alumno varón vs mujer & -1.915 & .917 & -2.089 & .038 & -3.719 & -.111 \\
\hline & Edad alumnado & -2.112 & .803 & -2.630 & .009 & -3.692 & -.532 \\
\hline
\end{tabular}

Se verifican los supuestos matemáticos del modelo con el test de Box (Box's $M=$ $10.757, F(6,6.49)=1.773, p=.1)$ para estos factores y el buen ajuste con el análisis de los residuos estandarizados mediante gráficas $Q-Q$.

Actitud: Incompetencia Social 
La Agresividad-terquedad, Dominancia, Apatía-retraimiento y Ansiedad-timidez aparecen como indicadores de la Incompetencia social de los niños. Para determinar la influencia de las variables independientes sobre ellos, y previamente comprobado el grado de correlación para el que el coeficiente de Pearson muestra unos valores que oscilan entre .1 y .5, se generó un modelo de regresión del que se excluyó el factor de Agresividad-terquedad, ya que ninguna de las variables independientes mostró relación con él. Los contrastes multivariados para el modelo final ponen de manifiesto que las variables independientes que tienen una influencia significativa sobre la Incompetencia social del alumnado son: el sexo $(V=.70, F(3$, $294)=7.346, p<.001)$ y edad del alumno $(V=.29, F(3,294)=2.96, p<.05)$; el sexo $(V=$ $.038, F(3,294)=3.821, p<.05)$ y la edad del profesor $(V=.05, F(3,294)=5.72, p<.001)$ así como el grado de HA de éste $(V=.032, F(3,294)=3.214, p<.05)$.

Al analizar su influencia sobre las variables dependientes consideradas encontramos, tal y como se puede ver en la Tabla 3, que los niños tienden a ser más Dominantes que las niñas y su grado de Apatía y retraimiento es también superior al de éstas. Apatía que se ve aumentada en 1.58 puntos por cada año más que va teniendo el alumnado. Del mismo modo se observa que cuando los alumnos pasan más tiempo con un profesor que es varón su puntuación en Dominancia y en Ansiedad-timidez es inferior a la puntuación media del alumnado que está con profesoras mujeres. Así mismo, a medida que aumenta la edad del docente mayor es el grado de Dominancia y de Apatía-retraimiento. Por último, cuanto mayor es la HA del profesorado menor es el grado de Dominancia del alumnado.

Tabla 3. Modelo de regresión para la Incompetencia Social

\begin{tabular}{|c|c|c|c|c|c|c|c|}
\hline \multirow[b]{2}{*}{ Variable depediente } & \multirow[b]{2}{*}{ Parámetros } & \multirow[b]{2}{*}{$\mathrm{B}$} & \multirow[b]{2}{*}{ Error típ. } & \multirow[b]{2}{*}{$\mathrm{t}$} & \multirow[b]{2}{*}{$\mathrm{p}$} & \multicolumn{2}{|c|}{$\begin{array}{c}95 \% \text { Intervalo de } \\
\text { confianza }\end{array}$} \\
\hline & & & & & & $\begin{array}{l}\text { Límite } \\
\text { inferior }\end{array}$ & $\begin{array}{l}\text { Límite } \\
\text { superior }\end{array}$ \\
\hline & Constante & 3.820 & 8.184 & .467 & .641 & -12.287 & 19.926 \\
\hline \multirow{5}{*}{ Dominancia } & Sexo alumno varón vs mujer & 2.504 & .750 & 3.340 & .001 & 1.029 & 3.980 \\
\hline & Sexo profesor varón vs mujer & -1.687 & .825 & -2.047 & .042 & -3.310 & -.065 \\
\hline & Edad alumnado & .901 & .650 & 1.385 & .167 & -.379 & 2.181 \\
\hline & Edad profesorado & .194 & .061 & 3.200 & .002 & .075 & .314 \\
\hline & HA profesorado & -.111 & .039 & -2.846 & .005 & -.187 & -.034 \\
\hline \multirow{6}{*}{$\begin{array}{l}\text { Apatía- } \\
\text { retraimiento }\end{array}$} & Constante & 1.163 & 9.951 & .117 & .907 & -18.421 & 20.748 \\
\hline & Sexo alumno varón vs mujer & 2.196 & .912 & 2.408 & .017 & .401 & 3.990 \\
\hline & Sexo profesor varón vs mujer & -.534 & 1.003 & -.533 & .595 & -2.507 & 1.439 \\
\hline & Edad alumnado & 1.588 & .791 & 2.008 & .046 & .032 & 3.144 \\
\hline & Edad profesorado & .221 & .074 & 2.995 & .003 & .076 & .367 \\
\hline & HA profesorado & -.072 & .047 & -1.525 & .128 & -.165 & .021 \\
\hline \multirow{3}{*}{$\begin{array}{l}\text { Ansiedad- } \\
\text { timidez }\end{array}$} & Constante & 32.197 & 10.148 & 3.173 & .002 & 12.227 & 52.168 \\
\hline & Sexo alumno varón vs mujer & -1.484 & .930 & -1.596 & .112 & -3.313 & .346 \\
\hline & Sexo profesor varón vs mujer & -2.652 & 1.022 & -2.594 & .010 & -4.664 & -.640 \\
\hline
\end{tabular}




\begin{tabular}{lrrrrrr} 
Edad alumnado & -.776 & .806 & -.963 & .337 & -2.363 & .811 \\
Edad profesorado & .146 & .075 & 1.944 & .053 & -.002 & .295 \\
HA profesorado & -.055 & .048 & -1.148 & .252 & -.150 & .040 \\
\hline
\end{tabular}

Igualmente se verifican los supuestos matemáticos del modelo con el test de Box (Box's $M=16.677, F(18,1.77 \mathrm{E} 05)=.908, p=.569)$ para estos factores y con el análisis de los residuos estandarizados mediante gráficas Q-Q, que ponen de manifiesto la tendencia normal de los datos.

Finalmente, para analizar la influencia de las variables del profesorado en la Cohesión de grupo y con el objetivo de evitar colinealidad en la modelización de los datos, al estar relacionadas la AA y la HA, se desarrollaron dos modelos diferentes, uno para cada una de ellas, ajustados por la edad y sexo del profesor. Los resultados obtenidos (Tabla 4) muestran que en ambos, la edad y el sexo influyen de manera significativa en la Cohesión del grupo. Es más probable que dicha Cohesión sea baja o muy baja cuando, por un lado, la edad del profesorado aumenta, de tal manera que el odds de que un grupo tenga una Cohesión buena es 1.038 (1/0.964) veces menor por cada año que aumente la edad del profesor $(O R=.964, p<.0001)$, y por otro cuando el profesor es varón $(O R=.001, p<.0001$ y $O R=.021, p<.0001)$.

En el modelo generado para la AA, se observa que a mayor AA en los varones existe mayor probabilidad de que la Cohesión del grupo sea buena $(O R=1.095, p<.0001)$. No obstante, cuando el sexo del profesorado es mujer, el hecho de que ésta sea más AA también aumenta la probabilidad de mayor Cohesión, aunque no de manera estadísticamente significativa. Con relación a la HA, se aprecia de nuevo que su aumento en los varones mejora significativamente la Cohesión del grupo, de manera que, a mayor HA más probable será que la Cohesión del grupo sea buena $(O R=1.072, p<.0001)$. Sin embargo, su incremento en las profesoras afecta negativamente a la Cohesión de grupo $(O R=.984 p=.024)$.

Tabla 4. Modelo de Estimación de Ecuaciones Generalizadas para la Cohesión

\begin{tabular}{|c|c|c|c|c|c|c|c|c|}
\hline \multirow[b]{2}{*}{ Parámetros } & \multirow[b]{2}{*}{ B } & \multirow[b]{2}{*}{ Error típ. } & \multicolumn{3}{|c|}{ Contraste de hipótesis } & \multirow{2}{*}{$\begin{array}{c}\text { OR } \\
\text { Epx(B) }\end{array}$} & \multicolumn{2}{|c|}{$\begin{array}{l}\text { 95\% Intervalo de } \\
\text { confianza para } \\
\text { Wald } \operatorname{Exp}(\mathrm{B})\end{array}$} \\
\hline & & & $\begin{array}{c}\text { Wald Chi- } \\
\text { Square }\end{array}$ & $\mathrm{df}$ & $\mathrm{p}$ & & $\begin{array}{l}\text { Límite } \\
\text { inferior }\end{array}$ & $\begin{array}{l}\text { Límite } \\
\text { superior }\end{array}$ \\
\hline \multicolumn{9}{|c|}{ Modelo GEE para la AA } \\
\hline Edad & -.037 & .005 & 53.425 & 1 & .000 & .964 & .955 & .974 \\
\hline Varón vs. mujer & -6.872 & .9607 & 51.173 & 1 & .000 & .001 & 0 & .007 \\
\hline AA & .009 & .0067 & 1.843 & 1 & .175 & 1.009 & .996 & 1.022 \\
\hline
\end{tabular}




\begin{tabular}{|c|c|c|c|c|c|c|c|c|}
\hline $\begin{array}{l}\text { Sexo*AA } \\
\text { Constante }\end{array}$ & $\begin{array}{r}.091 \\
1.182 \\
\end{array}$ & .0127 & 51.173 & 1 & .000 & 1.095 & 1.068 & 1.122 \\
\hline \multicolumn{9}{|c|}{ Modelo GEE para la HA } \\
\hline Edad & -.037 & .0048 & 60.324 & 1 & .000 & .964 & .955 & .973 \\
\hline Varón vs. mujer & -3.845 & .5462 & 49.554 & 1 & .000 & .021 & .007 & .062 \\
\hline HA & -.016 & .0072 & 5.104 & 1 & .024 & .984 & .97 & .998 \\
\hline Sexo*HA & .069 & .0091 & 57.696 & 1 & .000 & 1.072 & 1.053 & 1.091 \\
\hline Constante & -.007 & & & & & & & \\
\hline
\end{tabular}

En ambos modelos los residuos variaron entre - 2 y 2 indicando el buen ajuste del modelo y la idoneidad de las estimaciones según los datos observados.

\section{Discusión y Conclusiones}

La competencia social ha sido ampliamente estudiada desde la perspectiva del alumnado, sin embargo no lo ha sido tanto desde la relación profesor-alumno, más concretamente del papel que desempeña el docente en su desarrollo. Prueba de que esa correspondencia existe la encontramos en el estudio de Jennings y DiPrete (2010) que, sin mostrar los mecanismos por los que sucede, señalan que los profesores influyen en las habilidades sociales de los alumnos y que la variabilidad en su efecto reside en la importancia que los maestros otorgan a estas habilidades para el desarrollo del niño. Investigaciones realizadas con profesorado universitario han afirmado el vínculo entre la personalidad del docente y su capacidad para actuar correctamente en el aula, así como la influencia de la competencia social del profesor sobre la del alumnado (Vališová, 2011).

Los resultados del presente trabajo permiten extender estos hallazgos al ámbito de la Educación Primaria no sólo aportando evidencia de la presencia de ese vínculo relacional sino también de la relación entre las variables que lo configuran. Se ha podido constatar que la edad, el sexo, la autoasertividad, y la heteroasertividad del docente influyen en el desarrollo de las actitudes sociales de su alumnado.

Según muestran los datos, la conducta asertiva del maestro, globalmente considerada, no parece estar relacionada significativamente, con las actitudes sociales que los niños desarrollan. Sin embargo, al descomponer ambos constructos sólo se encuentra que la heteroasertividad se presenta como un factor amortiguador de la dominancia (actitud antisocial), o lo que es lo mismo, de la tendencia del alumnado a manipular y a aprovecharse de los otros en 
beneficio propio, sintiéndose superior a ellos. No se asume, pues, la hipótesis 7, referida a la autoasertividad, y se confirma parcialmente la 9, que propone que los maestros heteroasertivos frenan el desarrollo de actitudes antisociales y asociales en su alumnado. Estudios de espectro más amplio ya sugirieron la influencia de los aspectos socio-emocionales del maestro en el estudiante, a través de su práctica docente (Merchán y González, 2012).

Asimismo, los varones y la menor edad del profesor tienen un efecto significativo de disminución de la dominancia en el alumno; lo que nos lleva a no admitir las hipótesis 3 y 5. En éstas se afirma lo contrario: tanto ser varón como tener menor edad promueve el desarrollo de actitudes antisociales y asociales en los niños. Del mismo modo se rechazan las hipótesis 2, 4, 6 y 8, señalando que ninguna de las variables del docente consideradas en este trabajo (edad, sexo, autoasertividad y heteroasertividad) intervienen en el desarrollo de las actitudes prosociales.

Por otra parte, a nivel grupal, los resultados indicaron la existencia de una correspondencia entre los valores y actitudes sociales que los profesores tienen cuando interaccionan con sus alumnos y las actitudes sociales de éstos cuando se relacionan con los iguales. Esto es, los docentes asertivos generan una buena cohesión entre los miembros del grupo clase, en consonancia con lo establecido en la hipótesis 10. Esta observación es coherente con los hallazgos de Vališová (2011) que sostiene la influencia de la competencia social del docente sobre la del alumno en términos de su capacidad para manejar grupos de trabajo, comunicarse y cooperar con el grupo, discutir y negociar de modos no violentos, hacer frente a aspectos emocionales referentes a sí mismo y a los demás, o su habilidad para determinar las reglas que gobieran las interacciones de los estudiantes en el aula, así como las normas y el respeto recíproco entre ellos. Aspectos, todos ellos, que forman parte de la práctica de cualquier profesor, y que conllevan la creación de un clima de aula favorecedor del desarrollo de capacidades socio-emocionales (Merchán y González, 2012).

Con relación al grupo, nuestros datos muestran, igualmente, la influencia del género del maestro en los procesos y dinámicas sociales que ocurren en la clase. Así, la cohesión del grupo no se ve favorecida cuando el docente es varón y cuando aumenta su edad, aunque puede mejorarla optimizando tanto su autoasertividad como su heteroasertividad. Sin embargo, las profesoras con un elevado nivel de heteroasertividad tienden a disminuir esa cohesión o, en su caso, a no generarla. Estas diferencias relacionadas con el sexo pueden explicarse desde 
una doble vertiente. Por un lado, en términos de comportamientos asociados a estereotipos de género con relación al proceso de socialización de los sujetos. Mientras que a los niños se les fomenta más la agresividad y el comportamiento competitivo, a las niñas se las enseña a anteponer las necesidades ajenas y a inhibir los deseos por deferencia a los demás (Inglés et al., 2008). Incluso los costes sociales de la demostración de niveles bajos o altos de asertividad pueden ser más graves para las mujeres que para los hombres porque suponen una violación del rol del género femenino (Eagly y Karau, 2002). Por otro lado, con independencia del género, los estudios sobre el constructo de asertividad muestran una relación curvilínea entre estas variables, señalando que niveles extremos en la manifestación de la conducta asertiva dificultan las relaciones interpersonales (Ames y Flynn, 2007).

Por último, la conjetura de que un valor que forme parte de la escala de valores del docente parece contribuir a que el alumno lo haga suyo sin ser explícitamente enseñado, sólo observado y vivenciado, puede verse apoyada por los resultados de investigaciones como la realizada por Moraleda et al. (1998), Muñiz y Suárez (2012) o esta misma, al no confirmarse la hipótesis 1 , poniendo de manifiesto la irrelevancia de la titularidad del centro, público o concertado, para la formación en valores sociales de los alumnos. Lo que, a su vez, refuerza el concepto del "hábito del profesor isla" (Fernández, 1998, p.38) en la transmisión de estos valores.

Si bien este trabajo destaca por ser uno de los primeros en poner de relieve la influencia del docente en el desarrollo de las actitudes sociales de su alumnado, este mismo hecho supone una limitación al no poderse comparar y discutir los resultados con los obtenidos por estudios previos. Además, debemos ser cautos con lo que muestran los datos dada la limitada representatividad de la muestra.

A pesar de estas limitaciones, los resultados nos permiten concluir que, efectivamente, las interacciones que tienen lugar en el contexto educativo están mediadas por el hecho de que las personas se influyen mutuamente, y que el comportamiento asertivo del docente es uno de los componentes de la vida del aula que inciden en la configuración de las relaciones interpersonales del alumnado y, en último término, en la orientación de sus valores sociales. El maestro favorece así a su desarrollo personal y social. 
La asertividad se configura, por tanto, como una competencia interpersonal deseable en el perfil del buen docente ya que además, como cualquier otra habilidad de interacción social, contribuye al bienestar psicológico de profesores y alumnos así como a un aprendizaje de calidad (Talvio et al., 2013). Se plantea, pues, la necesidad de continuar indagando en este ámbito y de explorar la conveniencia de la formación personal del maestro. Aspecto éste que en el área de la educación emocional defienden con determinación autores como Extremera y Fernández (2004) y Merchán y González (2012)

Finalmente, poner en evidencia que los valores y actitudes sociales del docente influyen en los del alumnado tiene implicaciones tanto para la investigación en la enseñanza de valores sociales como en la de estrategias para la mejora de la convivencia y del clima social de clase. Se abre la posibilidad a que en los programas que pretenden modificar comportamientos abusivos de los niños, la intervención no se centre exclusivamente en los implicados sino que incluya el logro de una conducta asertiva ajustada por parte del docente.

\section{Referencias}

Adams, G. P., \& Roopnairene, J. L. (1994). Physical attractiveness, social skills, and samesex peer popularity. Journal of Group Psychotherapy, Psychodrama and Sociometry, $47,15-35$.

Allen, J.P., Weissberg, R.P., \& Hawkins, J.A. (1989). The relation between values and social competence in early adolescence. Developmental Psychology, 25(3), 458-464. Doi: $10.1037 / 0012-1649.25 .3 .458$

Ames, D.R., \& Flynn, F.J. (2007). What breaks a leader: The curvilinear relation between assertiveness and leadership. Journal of Personality and Social Psychology, 92(2), 307-324. Doi:10.1037/0022-3514.92.2.307

Barr, L.K., Kahn, J. H., \& Schneider, W.J. (2008). Individual differences in emotion expression: Hierarchical structure and relations with psychological distress. Journal of Social and Clinical Psychology, 27, 1045-1077. Doi: 10.1521/jscp.2008.27.10.1045

Blankemeyer, M., Flannery, D., \& Vazsonyi, A. (2002). The role of aggression and social competence in children's perceptions of the child-teacher relationship. Psychology in the Schools, 39 (3), 293-304. Doi: 10.1002/pits.10008 
Braithwaite, V., \& Blamey, R. (2001). Consenso, estabilidad y significado en los valores sociales abstractos. En M. Ros y V. Gouveia, (Eds.), Psicología Social de los Valores Humanos (pp. 153 - 172). Madrid: Biblioteca Nueva.

Bueno, M.R., \& Garrido, M. A. (2012). Relaciones interpersonales en la educación. Madrid: Pirámide.

Caballo, V. (2005). Manual de evaluación y entrenamiento en habilidades sociales. Madrid: Siglo XXI.

Cerezo, F. (2000). BULL-S. Test de Evaluación de la Agresividad entre Escolares. Bizkaia: ALBOR-COAHS.

Corapci, F. (2008). The role of child temperament on Head Start preschoolers' social competence in the context of cumulative risk. Journal of Applied Developmental Psychology, 29, 1-16. Doi:10.1016/j.appdev.2007.10.003

Corredor, G., Justicia-Arráez, A., Martínez, M. C., \& Justicia, F. (2013). Aprender a Convivir. Un programa para la mejora de la competencia social del alumnado de Educación Infantil y Primaria. Electronic Journal of Research in Educational Psychology, 11(31), 883-894. Doi: 10.14204/ejrep.31.13105

Delamater, R.J., \& Mcnamara, J.R. (1991). Perceptions of assertiveness by women involved in a conflict situation. Behaviour Modification, 15, 173-193. Doi: $10.1177 / 01454455910152004$

Delors, J., Amagi, I., Carneiro, R., Chung, F., Geremek, B., Gorham, W., ... \& Nanzhao, Z. (1996). La educación encierra un tesoro: informe para la UNESCO de la Comisión Internacional sobre la Educación para el Siglo Veintiuno. Madrid: UNESCO. Santillana

Delval, J., \& Enesco, I. (1994). Moral, Desarrollo y Educación. Madrid: ANAYA.

Díaz, A. (2006). La educación en valores: Avatares del currículum formal, oculto y temas transversales. Revista Electrónica de Investigación Educativa, 8 (1), 1-15.

Eagly, A.H., \& Karau, S.J. (2002). Role congruity theory of prejudice toward female leaders. Psychological Review, 109, 573-598.

Extremera, N., \& Fernández, P. (2004). La importancia de desarrollar la inteligencia emocional en el profesorado. Revista Ibero-Americana de Educación, 33, 1-10

Fernández, I. (1998). Prevención de la violencia y resolución de conflictos. El clima escolar como factor de calidad. Madrid: Narcea.

Field, A. (2005). Discovering Statistics Using SPSS. Londres: SAGE. 
Frey, K., Hirschstein, M., \& Guzzo, B. (2000). Second Step: preventing aggression by promoting social competence. Journal of Emotional and Behavioral Disorders, 8, 102112. Doi: $10.1177 / 106342660000800206$

García, M., \& Magaz, A. (2000). ADCAs. Autoinformes de Actitudes y Valores en las Interacciones Sociales. Bizkaia: ALBOR-COAHS.

García, F. J., Sureda, I., \& Monjas, M. I. (2010). El rechazo entre iguales en la educación primaria: Una panorámica general. Anales de Psicología, 26(1), 123-136.

Gismero, E. (1996). Habilidades sociales y anorexia nerviosa. Madrid: Publicaciones Universidad Pontificia.

Gómez, A. (2005). La enseñanza y el aprendizaje de los valores en la educación Deportiva. Revista Internacional de Medicina y Ciencias de la Actividad Física y el Deporte, 5(18), 89-99.

Hernández, C. (1986). Los valores sociales, un instrumento para el conocimiento social del niño. Su reflejo en la comunicación publicitaria. Infancia y Aprendizaje, (35-36), 109122.

Inglés, C.J., Martínez-Monteagudo, M.C., Delgado, B., Torregrosa, M.S., Redondo, J., Benavides, G., García-Fernández, J.M., \& García-López, L.J. (2008). Prevalencia de la conducta agresiva, conducta prosocial y ansiedad social en una muestra de adolescentes españoles: un estudio comparativo. Infancia y Aprendizaje, 31 (4), 449-461.

Jennings, J. L., \& Diprete, T. A. (2010). Teacher effects on social and behavioral skills in early elementary school. Sociology of Education, 83 (2), 135-159. Doi: $10.1177 / 0038040710368011$

Ladd, G .W. (1990). Having friends, keeping friends, making friends and being liked by peers in the classroom: predictors of children's early school adjustment? Child Development, 58, 306-315. Doi: 10.2307/1130877

Luengo, J.A. (2011). Ciberbulling. Guía de recursos para centros educativos en casos de ciberacoso. Madrid: Defensor del Menor en la Comunidad de Madrid.

Mcloughlin, C. (2009). Positive peer group interventions: An alternative to individualized interventions for promoting prosocial behavior in potentially disaffected youth. Electronic Journal of Research in Educational Psychology, 19, 7(3), 1131-1156.

Merchán, I. M. y González, J. D. (2012). Análisis de la eficacia de un Programa de Inteligencia Emocional con profesores de Badajoz y Castelo Branco. Campo Abierto, 31(1), 5168. 
Monjas, M. I., \& González, B. P. (Eds.) (1998). Las habilidades sociales en el currículo. Madrid: Centro de Investigación y Documentación Educativa.

Monjas, M. I. (2002). Programa de de enseñanza de habilidades de interacción social (PEHIS). Madrid: CEPE.

Moraleda, M., González, A., \& García-Gallo, J. (1998). AECS. Actitudes y Estrategias Cognitivas Sociales. Madrid: TEA.

Muñiz, M. A., \& Pérez, M. H. (2010). La educación en valores afectivos, comportamientos sociales y desarrollo personal: variables relevantes en el proceso formativo. (Consultado el 06/05/2013) http://2010.economicsofeducation.com/user/pdfsesiones/063.pdf

Muñiz, M. A., \& Suárez, J. (2012). Educación en valores y competencias sociales: Diferencias (o no) entre institutos públicos y colegios concertados. En A. Caparrós, (Ed.). Investigaciones de Economía de la Educación (pp. 208-222). Asociación de Economía de la Educación: E-books Investigaciones de Economía de la Educación. http//repec.economicsofeducation.com/2011/malaga/06-12.pdf

Muñoz, A., \& Jiménez, M. (1995). Relaciones interpersonales y clima social del aula. En M.V. Trianes (Ed.). Psicología de la Educación para profesores (pp. 261 - 278). Madrid: Pirámide.

Muñoz, J. M., Carreras, M. R., \& Braza, P. (2004). Aproximación al estudio de las actitudes y estrategias de pensamiento social y su relación con los comportamientos disruptivos en el aula en educación secundaria. Anales de Psicología, 20 (1), 81-91.

Ollendick, T. G., Francis, G., \& Baum, G. (1991). Sociometric status: Its stability and validity among neglectec, rejected and popular children. Journal of Child Psychology and Psychiatry, 32 (3), 525-534. Doi: 10.1111/j.1469-7610.1991.tb00329.x

Oñate, M.P. (1998). Procesos de grupo en el aula. En J.A. Bueno y C. Castanedo (Coords.). Psicología de la Educación Aplicada (pp. 543-568). Madrid: CCS

Ortega, P., \& Minguez, R. (2001). Los Valores en la Educación. Barcelona: Arial Educación.

Paniego, J. A. (1999). Cómo podemos educar en valores. Madrid: CCS.

Pérez, C. (2009). Valores y Normas para la Convivencia en el Aula. Madrid: EOS.

Pérez, I (2000). Habilidades sociales: educar hacia la autorregulación. Barcelona: Horsori.

Rivière, A. (1992). La Teoría cognitiva social del aprendizaje: Implicaciones educativas. En J. Coll, J. Palacios y A. Marchesi (Coords.). Desarrollo psicológico y educación, II. (pp. 69-80). Madrid: Alianza Psicología 
Ros, M. (2001a). Psicología social de los valores: una perspectiva histórica. En M. Ros y V. Gouveia (Ed.). Psicología Social de los Valores Humanos (pp. 27-51). Madrid: Biblioteca Nueva.

Ros, M. (2001b). Valores, actitudes y comportamiento: una nueva visita a un tema clásico. En M. Ros y V. Gouveia (Eds.). Psicología Social de los Valores Humanos (pp. 79-99). Madrid: Biblioteca Nueva.

Stevens, J. P. (2002). Applied multivariate statistics for the social sciences. Nueva York: Routledge Academic.

Talvio, M., Lonka, K., Komulainen, E., Kuusela, M., \& Lintunen, T. (2013). Una nueva mirada a la formación en eficacia docente de Gordon (TET): Un estudio-intervención en el aprendizaje social y emocional del profesorado. Electronic Journal of Research in Educational Psychology, 11(3), 693-716. Doi: 10.14204/ejrep.31.13073

Trianes, M.V. (1996). Educación y competencia social: un programa en la escuela. Málaga: Aljibe.

Trianes, M.V., De La Morena, M.L., \& Muñoz, A.M. (1999). Relaciones sociales y prevención de la inadaptación social y escolar. Málaga: Aljibe.

Twisk, L.W. (2007). Applied Longitudinal Data Analysis for Epidemiology: A Practical Guide. Cambridge: University Press.

Vališová, A. (2011). The Problem of the Social Competence of Teachers-Engineers. Studia z Teorii Wychowania : pótrocznik Zespotu Teorii Wychowania Komitetu Nauk Pedagogicznych PAN, 2/1 (2), 168-171 